University of Nebraska - Lincoln

DigitalCommons@University of Nebraska - Lincoln

CSE Conference and Workshop Papers

Computer Science and Engineering, Department

2005

\title{
Performance Analysis of Sparse Traffic Grooming in WDM Mesh Networks
}

Wang Yao

University of Nebraska-Lincoln, wyao@cse.unl.edu

Mengke Li

University of Nebraska-Lincoln, mli@cse.unl.edu

Byrav Ramamurthy

University of Nebraska-Lincoln, bramamurthy2@unl.edu

Follow this and additional works at: https://digitalcommons.unl.edu/cseconfwork

Part of the Computer Sciences Commons

Yao, Wang; Li, Mengke; and Ramamurthy, Byrav, "Performance Analysis of Sparse Traffic Grooming in WDM Mesh Networks" (2005). CSE Conference and Workshop Papers. 122.

https://digitalcommons.unl.edu/cseconfwork/122

This Article is brought to you for free and open access by the Computer Science and Engineering, Department of at DigitalCommons@University of Nebraska - Lincoln. It has been accepted for inclusion in CSE Conference and Workshop Papers by an authorized administrator of DigitalCommons@University of Nebraska - Lincoln. 


\title{
Performance Analysis of Sparse Traffic Grooming in WDM Mesh Networks
}

\author{
Wang Yao, Mengke Li and Byrav Ramamurthy \\ Department of Computer Science and Engineering, \\ University of Nebraska-Lincoln, Lincoln NE 68588 USA \\ Email: \{wyao,mli, byrav\}@cse.unl.edu
}

\begin{abstract}
Sparse traffic grooming is a practical problem to be addressed in heterogeneous multi-vendor optical WDM networks where only some of the optical cross-connects (OXCs) have grooming capabilities. Such a network is called as a sparse grooming network. The sparse grooming problem under dynamic traffic in optical WDM mesh networks is a relatively unexplored problem. In this work, we propose the maximize-lightpathsharing multi-hop (MLS-MH) grooming algorithm to support dynamic traffic grooming in sparse grooming networks. We also present an analytical model to evaluate the blocking performance of the MLS-MH algorithm. Simulation results show that MLSMH outperforms an existing grooming algorithm, the shortestpath single-hop (SPSH) algorithm. The numerical results from analysis show that it matches closely with the simulation. The effect of the number of grooming nodes in the network on the blocking performance is also analyzed.
\end{abstract}

Index Terms-Sparse traffic grooming, blocking probability, performance analysis, wavelength-division multiplexing.

\section{INTRODUCTION}

Wavelength division multiplexing (WDM) based optical networking architectures using optical cross-connects (OXCs) are promising solutions to the next-generation long-haul transport networks. The transmission rate on a wavelength channel has reached OC-192 (10Gbps) and is expected to reach OC-768 (40Gbps) in the future. While WDM technology enables huge amounts of bandwidth, the traffic demand is increasing at an explosive rate as well. The traffic demand granularity varies a lot, possibly from OC-3 (155Mbps) to OC-192 (10Gbps). The routing problem with the bandwidth gap between the low-rate connection and highrate wavelength channels is addressed as a two-layer traffic grooming problem [1]-[2] with the goal to effectively share resources in the optical networks.

The two layers involved in traffic grooming are the optical layer and the electronic layer. The optical layer is composed of OXCs with point-to-point fiber links connecting them. The OXC nodes and the fiber links constitute the physical topology of an optical network. The optical layer establishes lightpaths along wavelength channels within the fibers. All the lightpaths and their corresponding end nodes constitute the virtual (logical) topology. The electronic layer establishes traffic connections on top of the virtual topology. Fig. 1 shows the architecture of an $\mathrm{OXC}$ with grooming capability (G-OXC). The G-OXC has two switching fabrics. One is the wavelength switching fabric (WXC) which is capable of switching

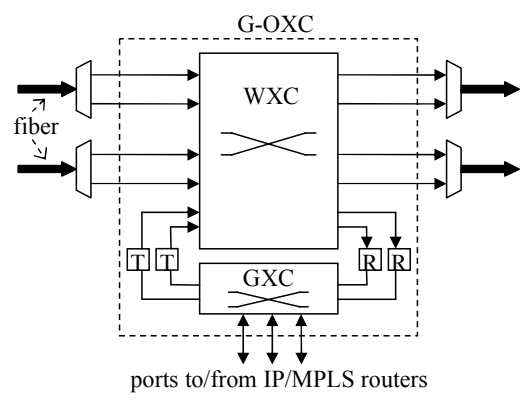

Fig. 1. The sample architecture of a G-OXC.

wavelengths in the optical domain. The other is the grooming fabric (GXC) which is capable of adding/dropping traffic streams to/from a wavelength in the electronic domain. Note that a lightpath only enters electronic domain (GXC) at its source node and destination node. The corresponding wavelength is bypassed in the WXCs at all the intermediate nodes of the lightpath.

As high-speed electronic equipments are still quite expensive, not all OXCs have the GXC components. An OXC without GXC is called a NG-OXC. In a heterogeneous multivendor optical network, it is not unusual that some nodes (Gnodes) are deployed with G-OXCs and the other nodes (NGnodes) are deployed with NG-OXCs. Such a network is called a sparse grooming network. If all the nodes of a network are NG-nodes, the network is called a dedicated grooming network. If all the nodes of a network are G-nodes, the network is called a full grooming network.

In a sparse grooming network, lightpaths can be divided into four categories, depending on the grooming capability of the end nodes. As shown in Fig. 2, the end-node-limited lightpath (EL-lightpath) is established between two NG-nodes. Only connections belonging to the same source-destination as the lightpath can be groomed on this lightpath. The lightpath established from an NG-node to a G-node is a source-limited lightpath (SL-lightpath). The connections that can be groomed on a SL-lightpath must originate from the same source as the lightpath but not necessarily be destined to the same destination. For a destination-limited lightpath (DL-lightpath) established from a G-node to an NG-node, all the connections that can be groomed on it must be destined to the same nodes as the lightpath, though they are not necessarily from the same source. A full lightpath established between two G-nodes has the most flexibility to share bandwidth among connections, because any connection can be groomed on it. 


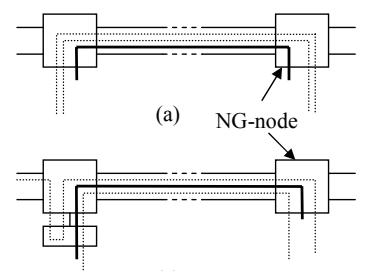

(c)

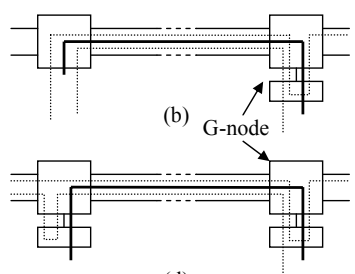

(d)
Lightpath

Connection

Fig. 2. The illustration of lightpath categories. (a) End-node-limited Lightpath, (b) Source-limited Lightpath, (c) Destination-limited Lightpath, (d) Full Lightpath.

In a dedicated grooming network, only EL-lightpaths can be established. Therefore, a connection can only use a single lightpath which has the same source/destination as the connection, and this is called single-hop traffic grooming. On the contrary, a full grooming network allows a lightpath to be used by connections from any source/destinations. Therefore, a connection may traverse multiple lightpaths, which is called as multi-hop traffic grooming.

Most previous work assume either a dedicated-grooming network or a full grooming network. The work in [2]-[3] studied online heuristic algorithms for dynamic traffic grooming in full grooming networks. The work in [4]-[5] proposed auxiliary graph models for traffic grooming in full grooming networks. The work in [6]-[8] present analytical models to evaluate the blocking performance of single-hop traffic grooming in dedicated grooming networks. The work in [9] presents an analytical model to evaluate the blocking performance of multi-hop traffic grooming in full grooming networks. The work in [10] is the only one that addresses sparse grooming networks. It presents an integer linear programming (ILP) formulation and several heuristics to solve the G-node selection problem in sparse grooming networks under static traffic.

In this work, we study the dynamic traffic grooming problem in sparse grooming networks. To efficiently exploit the sparse grooming capabilities in the network, we propose the maximize-lightpath-sharing multi-hop (MLS-MH) grooming heuristic to support the provisioning of multigranularity sub-wavelength connections in optical WDM mesh networks. An analytical model is presented to evaluate the connection blocking probability of the proposed sparse grooming algorithm. To the best of our knowledge, no previous work has addressed this issue in the literature.

The rest of the paper is organized as follows. Section II presents an online grooming algorithm for sparse grooming networks. Section III presents the analytical model to evaluate the blocking probability of the sparse grooming algorithm. Section IV presents the numerical results from simulation and analysis. Section V concludes the paper.

\section{GROOMING ALGORITHM}

Given the network resources, the objective of the dynamic traffic grooming problem is to maximize network throughput, or minimize connection blocking probability. One way to achieve this objective is to make efficient use of the wavelength bandwidth. However, an EL-lightpath only allows connections with the same source-destination to use its bandwidth. This constraint may reduce the utilization of the wavelength bandwidth if the connections from the same source-destination only use a small portion of the bandwidth. On the other hand, a full-lightpath allows connections from any source-destination to use its bandwidth. The SL-lightpath and the DL-lightpath are in between the EL-lightpath and the full-lightpath in terms of bandwidth sharing flexibility. Fig. 3 (a) shows a path with four nodes. Nodes 0 and 3 are NGnodes. Nodes 1 and 2 are G-nodes. If a lightpath $0-3$ is established on the path, only connections between nodes 0 and 3 can use it. However, if three lightpaths $0-1,1-2$ and 2-3 are established on the path, the bandwidth on three wavelength links can be used by more connections because the three lightpaths are SL-lightpath, full-lightpath and DL-lightpath respectively.

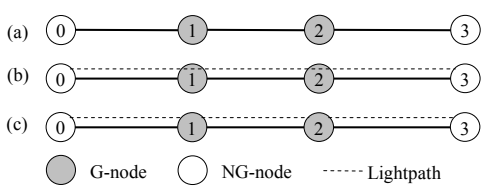

Fig. 3. (a) a path with four nodes, (b) an EL-lightpath $0-3$ on the path $0-1-2-3$, (c) three lightpaths $0-1,1-2$ and 2-3.

In this section, we propose the maximize-lightpath-sharing multi-hop (MLS-MH) grooming heuristic to provision dynamically arriving multi-granularity connections in sparse grooming networks. Let $\Phi(s, d, x)$ represents a connection request, where $s$ and $d$ are the source and the destination and $x$ is the bandwidth of the request. Fig. 4 shows the MLS-MH algorithm.

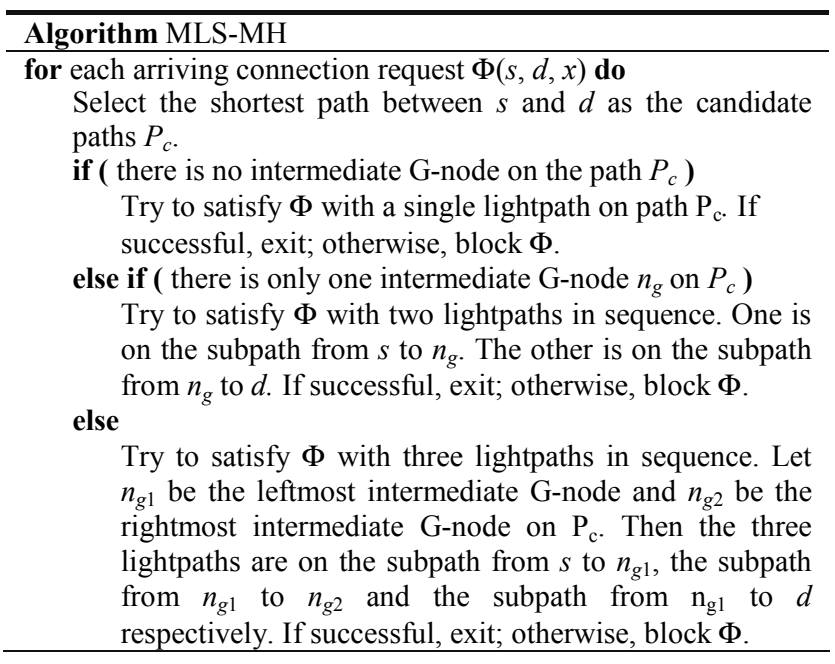

Fig. 4. The MLS-MH grooming algorithm.

\section{ANALYTICAL MODEL}

In this section, we present an analytical model to calculate the blocking probability of the MLS-MH grooming algorithm with random wavelength assignment.

The physical topology of a WDM optical network consists of $N$ nodes (labeled $1,2, \ldots, N$ ) and $J$ unidirectional fiber 
links (labeled 1, 2, ., J ). Each fiber link supports $W$ wavelengths and each wavelength has a normalized capacity $C$ which is the bandwidth of a wavelength divided by the smallest grooming granularity. In this paper, each unit capacity in a wavelength is called a channel (or time slot). We assume that there are enough grooming capacities (transceivers) in the network.

A connection request is denoted by $(s, d, x), s, d \in N$, $x \in X$, where $X$ is the set of the connection rates (granularities) supported in the network. To be consistent with the wavelength capacity unit, the connection rates are also normalized. For example, if the connections rates are $\{O C-3$, $O C-12, O C-48\}$ and the smallest grooming granularity is $O C$ 3 , then $X=\{1,4,16\}$. We assume the arrival of connections at rate $x$ for the node pair $(s, d)$ is a Poisson process with rate $\lambda_{s, d}^{x}$. The service time of the connection is exponentially distributed with unit mean.

Let $p_{s, d}$ represent the candidate path for connections between $(s, d)$. Let $l_{m, n}$ represent the path on which the lightpaths from node $m$ to node $n$ are established. In this section, we use the word "lightpath" to refer to the path on which real lightpaths are established. To denote that a lightpath $l$ is a sub-path (logical hop) of a path $p$, we use $l \in p$. To denote that a link $j$ is a hop of a lightpath $l$, we use $j \in l$.

\section{A. Traffic Model}

The offered traffic at rate $x$ to the candidate path $p_{s, d}$ is:

$A_{x}\left(p_{s, d}\right)=\lambda_{s, d}^{x}, \forall s, d, x$.

The offered traffic at rate $x$ on the lightpath $l$ due to the traffic on the path $p$ is:

$$
\begin{aligned}
A_{x}(l, p) & =A_{x}(p) \prod_{\forall l^{\prime}: l^{\prime} \in p-\{l\}}\left(1-B_{x}\left(l^{\prime}\right)\right) \\
& =A_{x}(p) \frac{1-B_{x}(p)}{1-B_{x}(l)}, \forall l, p .
\end{aligned}
$$

The offered traffic at rate $x$ on a lightpath $l$ is the sum of the traffic from all the paths which include $l$ as a hop.

$$
A_{x}(l)=\sum_{\forall p: l \in r} A_{x}(l, p), \forall l \text {. }
$$

The offered traffic at rate $x$ on the link $j$ is the sum of the traffic from all the lightpaths which include $j$ as a hop.

$$
A_{x}(j)=\sum_{\forall l: j \in l} A_{x}(l), \forall j .
$$

The offered traffic of the link $j$ is evenly distributed to each of its wavelengths because of the random wavelength assignment assumption. The offered traffic at rate $x$ on a single wavelength link $j$ is:

$$
A_{x}^{S W L}(j)=A_{x}(j) / W, \forall j \text {. }
$$

\section{B. Single Wavelength Link Model}

A single wavelength within a link is called as a single wavelength link (SWL). Equation (5) gives the arrival rates of connections at different granularities on a single wavelength link $j$. An SWL with multi-rate arrivals can be modeled as a multi-dimensional Markov Chain (MDMC). The state of the MDMC is a multi-dimensional vector $n=\left(n_{x}, x \in X\right)$, where $n_{x}$ is the number of connections at rate $x$ that are using the SWL. As each SWL has a total of $C$ channels, the state space $(\Omega)$ of the MDMC is constrained in (6).

$\left.\Omega=\left\{n: \sum_{x \in X} n_{x} x \leq C\right\}\right\}$.

Let $c_{F R E E}(n)$ be the number of free channels when the SWL is in state $n$. $c_{\text {FREE }}(n)$ can be calculated as follows:

$c_{\text {FREE }}(n)=C-\sum_{x \in X} n_{x} x$.

The equilibrium probability $\pi(n)$ of the MDMC being in state $n$ is given by

$\pi(n)=\frac{1}{G} \prod_{x \in X} \frac{\rho_{x}^{n_{x}}}{n_{x} !}, \forall n \in \Omega$,

where $\rho_{x}=A_{x}^{S W L}$ is the traffic load due to connections at rate $x$ and

$G=\sum_{n \in \Omega} \prod_{x \in X} \frac{\rho_{x}^{n_{x}}}{n_{x} !}$,

is the normalization constant [11].

Let $q(u)$ be the probability that there are $u$ free channels on the SWL. Then $q(u)$ is calculated as (10).

$q(u)=\sum_{\forall n: c_{F R E E}(n)=u} \pi(n), 0 \leq u \leq C$.

\section{Lightpath Blocking Probability Analysis}

In this paper, we assume that the WXCs within OXCs do not have wavelength conversion capabilities. Therefore, a lightpath is subject to the wavelength continuity constraint which stipulates that a lightpath must use the same wavelength along its path. In addition, a connection is subject to the channel continuity constraint which states that a connection must use the same channels within all the wavelength links of a lightpath. This is because the wavelength within a lightpath is bypassed in the WXCs at the intermediate OXCs without entering the electronic domain. Even with optical wavelength conversion capability, the channel continuity constraint remains valid. However, when $\mathrm{O} / \mathrm{E} / \mathrm{O}$ WXCs are used in the OXCs, the channel continuity constraint can be relaxed because wavelengths enter electronic domain at the intermediate OXCs of a lightpath.

Fig. 5 illustrates the wavelength continuity constraint and the channel continuity constraint within a path with three links, each with three wavelengths. Because of the wavelength continuity constraint, a path with W wavelengths is divided into $W$ single wavelength paths (SWPs). Each SWP consists of a sequence of SWLs. With random wavelength assignment, 
we further assume that the blocking on SWPs of different wavelengths is independent. Let $B_{x}^{S W P}(l)$ be the blocking probability of a connection at rate $x$ on a SWP of lightpath $l$. Then the blocking probability $B_{x}(l)$ of a connection at rate $x$ on the lightpath $l$ can be calculated as follows:

$$
B_{x}(l)=\left[B_{x}^{S W P}(l)\right]^{W}, \forall l .
$$

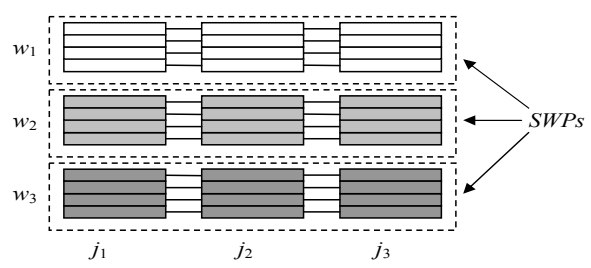

Fig. 5. A path with three wavelengths $w_{1}, w_{2}, w_{3}$ and three links $j_{1}, j_{2}, j_{3}$. Each wavelength has four channels $c_{1}, c_{2}, c_{3}$ and $c_{4}$. Channels within a wavelength are not interchangeable at OXCs. No wavelength conversion is allowed.

\section{Single Wavelength Path Blocking Probability Analysis}

Due to the channel continuity constraint, a connection can only use the channels which are free on all the SWLs within a SWP. We first analyze a two-hop SWP with two SWLs $j_{1}$ and $j_{2}$. Given that the numbers of free channels on $l_{1}$ and $l_{2}$ are $u_{1}$ and $u_{2}$ respectively, let $p_{v}\left(u_{1}, u_{2}\right)$ denote the probability that there are $v$ common free channels on the two-hop SWP. Then $p_{v}\left(u_{1}, u_{2}\right)$ can be calculated as:

$p_{v}\left(u_{1}, u_{2}\right)=\left\{\begin{array}{cc}\beta\left(u_{1}, u_{2}, v\right) & \text { if } 1 \leq u_{1}, u_{2} \leq C \text { and } \\ 0 & u_{1}+u_{2}-C \leq v \leq \min \left(u_{1}, u_{2}\right), \\ 0 & \text { otherwise }\end{array}\right.$

where

$\beta\left(u_{1}, u_{2}, v\right)=\frac{\left(\begin{array}{l}u_{1} \\ v\end{array}\right)\left(\begin{array}{l}C-u_{1} \\ u_{2}-v\end{array}\right)}{\left(\begin{array}{c}C \\ u_{2}\end{array}\right)}$.

For an $H$-hop SWP $(H \geq 3)$, given that $u_{j}$ is the number of free channels on the hop $i(1 \leq i \leq H)$, let $p_{v}\left(u_{1}, \ldots, u_{H}\right)$ be the probability that there are $v$ common free channels on the path. Then $p_{v}\left(u_{1}, \ldots, u_{H}\right)$ can be recursively calculated as:

$p_{v}\left(u_{1}, \ldots, u_{h}\right)=\sum_{k=v}^{u_{h-1}} p_{k}\left(u_{1}, \ldots, u_{h-1}\right) p_{v}\left(k, u_{h}\right)$, for $3 \leq h \leq H$.

For an H-hop SWP $(\mathrm{H} \geq 3)$, let $q_{i}(u)$ be the probability that there are $u$ free channels on the hop $i$. Let $q\left(u_{1}, \ldots, u_{H}\right)$ be the probability that there are $u_{i}$ free channels on the hop $i(1 \leq i \leq$ $\mathrm{H})$ of the SWP. We assume that the channel distribution on each link is independent. Therefore, $q\left(u_{1}, \ldots, u_{H}\right)$ can be calculated as follows:

$q\left(u_{1}, \ldots, u_{H}\right)=\prod_{1 \leq i \leq H} q_{i}\left(u_{i}\right)$.

A connection is blocked on a SWP of a lightpath $l$ if there are not enough common free channels on the SWP. Let $h(l)$ be the number of hops for a lightpath $l$, then
$B_{x}^{S W P}(l)=$

$\left\{\begin{array}{ll}\sum_{u_{1}=0}^{x-1} q\left(u_{1}\right) & \text { if } h(l)=1 \\ \sum_{u_{1}=0}^{C} \ldots \sum_{u_{H}=0}^{C} q\left(u_{1}, \ldots, u_{H}\right) \sum_{v=0}^{x-1} p_{v}\left(u_{1}, \ldots, u_{H}\right) & \text { if } h(l) \geq 2\end{array}, \forall l(16)\right.$

\section{E. Path Blocking Probability Analysis}

Let $B_{x}(p)$ be the probability of blocking a connection at rate $x$ on the path $p$. A connection is blocked on a path $p$ if it is blocked on any lightpath included in the path $p$.

$B_{x}(p)=1-\prod_{\forall l: l \in p}\left(1-B_{x}(l)\right), \forall p$.

\section{F. Network Blocking Probability Analysis}

The overall network blocking probability is the average blocking probability of all connections.

$$
B_{A V G}=\frac{\sum_{s, d} \sum_{x} x \times \lambda_{s, d}^{x} \times B_{x}\left(p_{s, d}\right)}{\sum_{s, d} \sum_{x} x \times \lambda_{s, d}^{x}} .
$$

\section{NumericAl Results AND Discussion}

To evaluate the performance of the MLS-MH grooming algorithm, we simulate it on the 14-node NSF network. For each node pair, only the shortest path is considered as the candidate path. We assume that there are 16 wavelengths on each fiber and there are enough transceivers in the network. The G-nodes and NG-nodes in the network are randomly selected. The symbol $\mathrm{G}$ represents the number of G-nodes in the network. For comparison reasons, we also simulate the shortest path single hop (SPSH) grooming algorithm. SPSH is the simplest and most popular grooming algorithm from the literature which uses a single lightpath on the shortest path to carry a connection. We also verify the proposed analytical model by comparing the analytical results with simulation results. Erlang fixed-point approximation method [12] is used to obtain the blocking probability from analytical model.

The following assumptions are used in our simulation and analysis. The arrival of connection requests on each node is a Poisson process with rate $\lambda$. The connection requests arriving at a node are uniformly destined to all the other nodes. The connection service time is exponentially distributed with unit mean. The grooming factor $C$ is 16 and the set of connection rates is $X=\{1,4,16\}$ after normalization. Connections at each rate request the same amount of total bandwidth. We simulate $1,000,000$ connection requests for each scenario in this section.

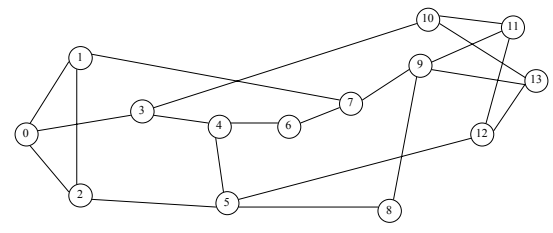

Fig. 6. The NSF Networks used in simulations and analysis. 


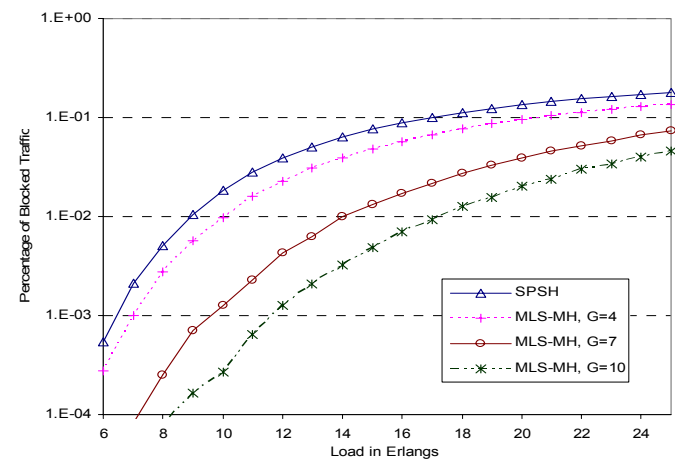

Fig. 7. Performance of grooming algorithms by simulations.

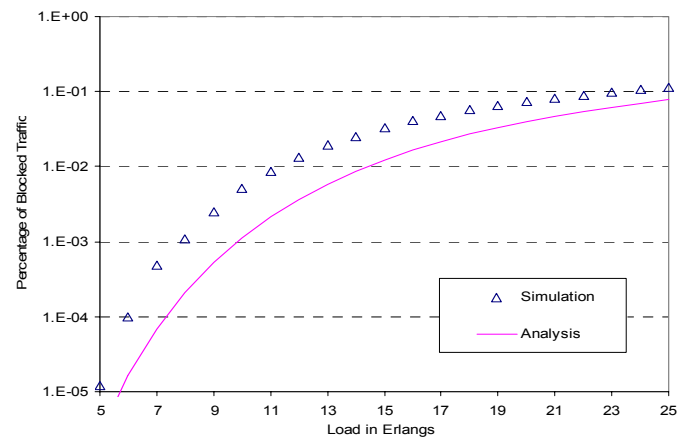

Fig. 8. The performance of MLS-MH vs. the load. $\mathrm{G}=6$.

Fig. 7 shows the blocking performance of the SPSH and MLS-MH grooming algorithms. As can be seen, the number of G-nodes (G) does not affect the performance of SPSH at all. This is because SPSH is a single-hop grooming algorithm which uses a single lightpath from the source to the destination to carry a connection. On the other hand, MLS$\mathrm{MH}$ outperforms SPSH and its performance generally improves as $\mathrm{G}$ increases. This result shows that a properly designed algorithm like MLS-MH is needed to exploit the grooming capability within a network.

To verify the correctness of the analytical model, we compare the analytical results with the simulation results in Fig. 8. The six G-nodes are randomly selected. As can be seen, the network blocking probabilities obtained from the analytical model match closely with those from simulations, although the analysis seems to slightly underestimate the network blocking probability.

Fig. 9 illustrates the performance of the MLS-MH algorithm in terms of network blocking probability when $G$ varies. As can be seen, the analytical model accurately depicts the trend line of the network blocking probability when $\mathrm{G}$ varies from 0 to 14 . This is an attractive property of the analytical model because we may use it in the design of a sparse grooming network. In that case, the analytical model can be used to estimate the network blocking probabilities of different design schemes with different number of grooming nodes, which should give the decision-maker a good picture of the tradeoff between the blocking performance and the cost (a function of G).

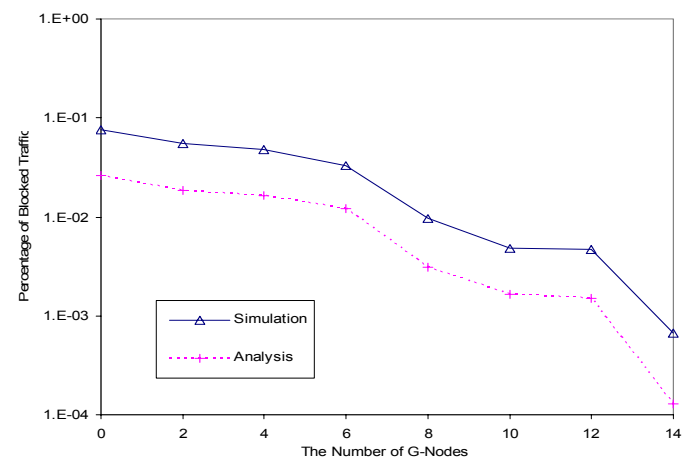

Fig. 9. The performance of MLS-MH vs. G. Load in Erlangs $=15$.

Intuitively, the network blocking probability should decrease as $\mathrm{G}$ increases. Although this is true for the NSF network as shown in Fig. 9, this may not always hold. In addition to $\mathrm{G}$, other factors such as the network topology, the grooming algorithm and the location of the G-nodes may also affect the blocking performance. As this work uses a random method to select G-nodes, intelligent placement of G-nodes in the network will be an interesting problem for future study.

\section{REFERENCES}

[1] A. Lardies, R. Gupta, R. A. Patterson, "Traffic grooming in a multi-layer network," Optical Networks Magazine, May 2001.

[2] K. Zhu, B. Mukherjee, "On-Line Approaches for Provisioning Connections of Different Bandwidth Granularities in WDM Mesh Networks," OFC 2002, pp.549-551, March 2002.

[3] C. Xin, Y. Ye, S. Dixit, C. Qiao, "An Integrated Lightpath Provisioning Approach in Mesh Optical Networks," OFC 2002, pp. 547-549, 2002.

[4] H. Zhu, H. Zang, K. Zhu, B. Mukherjee, "Dynamic Traffic Grooming in WDM Mesh Networks Using a Novel Graph Model," in Proc. IEEE Globecom 2002, vol. 3, pp. 2681-2685, Nov. 2002.

[5] W. Yao, B. Ramamurthy, "Constrained Dynamic Traffic Grooming in Optical WDM Mesh Networks with Link Bundled Auxiliary Graph Model," in Proc. IEEE Workshop on High Performance Switching and Routing 2004, pp. 287-291, Phoenix, Arizona, Apr. 2004.

[6] S. Thiagarajan and A. K. Somani, "A Capacity Correlation Model for WDM Networks with Constrained Grooming Capabilities," in Proc. IEEE ICC'01, vol. 5, pp. 1592 - 1596, Jun. 2001.

[7] R. Srinivasan and A. K. Somani, "Analysis of Multi-rate Traffic in WDM Grooming Networks," in Proc. ICCCN'02, pp. 296-301, Oct. 2002.

[8] C. Xin, C. Qiao, S. Dixit, "Traffic Grooming in Mesh WDM Optical Networks Performance Analysis," in Proc. IEEE GLOBECOM'03, vol. 7, pp. 3732-3736, Dec. 2003.

[9] C. Xin, and C. Qiao, "Performance Analysis of Multi-Hop Traffic Grooming in Mesh WDM Optical Networks," in Proc. IEEE ICC'03, pp. 237-242, Oct. 2003.

[10] K. Zhu, H. Zang, B. Mukherjee, "Design of WDM Mesh Networks with Sparse Grooming Capability," in Proc. IEEE Globecom 2002, vol. 3, pp. 2696-2700, Nov. 2002.

[11] D. Bertsekas and R. Gallager, Data Networks, Prentice Hall, 1992.

[12] A. Birman, "Computing approximate blocking probabilities for a class of all-optical networks," IEEE J. Select. Areas Commun., vol. 14, no. 5, pp. 852-857, Jun. 1996. 